\title{
Chemotherapy-related subjective cognitive impairment in breast cancer patients in semi-rural South Africa
}

\section{Authors:}

Nicholas M. Keetile ${ }^{1}$

Elzbieta Osuch ${ }^{1}$ (1)

Antonio G. Lentoor ${ }^{2}$

Affiliations:

${ }^{1}$ Department of

Pharmacology, School of Medicine, Sefako Makgatho

Health Sciences University,

Pretoria, South Africa

${ }^{2}$ Department of Clinical

Psychology, School of Medicine, Sefako Makgatho

Health Sciences University,

Pretoria, South Africa

Corresponding author:

Nicholas Keetile,

keetmn@gmail.co.za

Dates:

Received: 10 Jan. 2021

Accepted: 26 May 2021

Published: 26 July 2021

How to cite this article: Keetile, N.M., Osuch, E. \& Lentoor, A.G., 2021,

'Chemotherapy-related

subjective cognitive

impairment in breast cancer

patients in semi-rural South

Africa', Health SA Gesondheid

26(0), a1605. https://doi.

org/10.4102/hsag.v26i0.1605

\section{Copyright:}

(C) 2021 The Authors

Licensee: AOSIS. This work

is licensed under the

Creative Commons

Attribution License.

\section{Read online:}

Scan this QR code with your smart phone or mobile device to read online.
Background: Breast cancer is the most commonly diagnosed cancer amongst women worldwide. Whilst current evidence indicates the therapeutic benefits from the use of chemotherapy, self-perceived cognitive difficulties emerged as a frequent occurrence during and after chemotherapy treatment in breast cancer patients.

Aim: The current study sought to investigate self-perceived cognitive impairment in a group of breast cancer patients in semi-rural South Africa.

Setting: The patients were recruited from an outpatient oncology clinic at a semi-rural, tertiary academic hospital in Gauteng, South Africa.

Methods: In a randomised, quantitative, time-based series study, 30 female patients aged 21-60 years (mean age $=50$ years) diagnosed with stages II and III breast cancer on CMF (cyclophosphamide, methotrexate, fluorouracil) $(n=10)$ and FAC (fluorouracil, adriamycin, cyclophosphamide) $(n=20)$ chemotherapy regimens, completed the self-reported Functional Assessment of Cancer Therapy-Cognition (Fact-Cog) test as a measure of subjective cognitive functioning at three points during the course of treatment $\left(\mathrm{T}_{0}, \mathrm{~T}_{1}, \mathrm{~T}_{2}\right)$.

Results: The results of the paired sample $t$-tests showed the scores on the Fact-Cog test confirmed significant cognitive decline for both treatment groups from baseline $\left(\mathrm{T}_{0}\right)$ to completion $\left(\mathrm{T}_{2}\right)$ of chemotherapy; CMF group, $t(9)=2.91, p=0.017$ and the FAC group $t(19)=4.66, p<0.001$.

Conclusion: This study confirms that self-reported subjective cognitive impairment is common in breast cancer patients who received chemotherapy in a sample of South African patients. The results have implications for the overall care of cancer patients.

Contribution: The context-based knowledge engendered by the current study is expected to augment the continuum of care for breast cancer patients.

Keywords: breast cancer; adjuvant chemotherapy; Fact-Cog test; perceived cognitive impairment; chemotherapy-related cognitive impairment (CRCI); chemobrain.

\section{Introduction}

Mounting evidence suggests that breast cancer is the most commonly diagnosed cancer amongst women worldwide (DeSantis et al. 2015), accounting for $11.6 \%$ of all cancers globally (Bray et al. 2018). Despite projections for a substantial increase in new cases, the survival rates of patients with breast cancer have shown an enormous improvement since 1989, and this is attributed to early detection of the disease and the availability of effective treatment modalities (Akram et al. 2017). For example, one study showed that the 5-year survival of breast cancer in 17 countries across the world increased to $85 \%$ (Allemani et al. 2018).

Globally, standard doses of an adjuvant chemotherapy regimen comprising of cyclophosphamide, methotrexate, fluorouracil (CMF) and that composed of fluorouracil, adriamycin, cyclophosphamide (FAC) have improved survival rates of breast cancer patients over time, especially in countries of low and middle income (Isakoff 2010; Verrill 2009). Current research across continents indicates that the therapeutic benefits derived from the use of chemotherapy for breast cancer have been accompanied by debilitating cognitive impairment (Loh et al. 2016; Weiss, Walker \& Wiernik 2010). This condition (commonly referred to as 'chemobrain') manifests as diminished memory, concentration, attention and executive function (Ono et al. 2015; Simó et al. 2013) and its symptoms are detected in up to 75\% of patients during chemotherapy. These symptoms can persist for years in up to $35 \%$ of these patients post-treatment (Janelsins et al. 2012; Vardy et al. 2008). Cancer, age, fatigue, anxiety, depression and hormonal therapy have been highlighted in research as confounding factors for cognitive impairment and 
they can diminish the patients' quality of life (Munir et al. 2010; Selamat et al. 2014; Von Ah et al. 2013).

Most of the studies relating to chemobrain in patients with breast cancer were conducted amongst women in the United States of America (USA), Europe, Canada or Australia (Ribi 2012). This quantitative time-series thus sought to investigate the subjective cognitive function before, during and after chemotherapy in a group of female breast cancer patients in South Africa as measured on the Functional Assessment of Cancer Therapy-Cognition test (Fact-Cog Test). The significance of the study is that it will generate knowledge relevant to the local context with implication for the overall care and management of breast cancer patients.

\section{Materials and methods \\ Research design}

In a randomised, quantitative, time-series study, 30 out of 34 female patients with a breast cancer diagnosis prior to starting chemotherapy were included. Participants were aged 21-60 years at the time of recruitment. From the 34 patients, 4 were excluded ( 1 had previous chemotherapy exposure whilst 3 were over 60 years of age). All the participants were recruited from an outpatient oncology clinic at a semi-rural, tertiary hospital in Gauteng, during the period of October 2018 to October 2019 with the assistance of the treating oncologist, after confirming the stage of the patients' breast cancer.

\section{Study measures}

\section{Participants' health information}

Patients diagnosed with stages II and III breast cancer who met inclusion criteria (not undergoing concurrent radiation therapy and hormonal therapy, non-fluency in English, no concurrent psychotic disorder, epilepsy and dementia) were considered eligible for inclusion in the study. After diagnosis, patients were assigned to receive either CMF or FAC according to the following schedules: $\mathrm{CMF}$ (cyclophosphamide $100 \mathrm{mg} / \mathrm{m}^{2}$ orally, methotrexate $40 \mathrm{mg} / \mathrm{m}^{2}$ intravenously, 5-fluorouracil $600 \mathrm{mg} / \mathrm{m}^{2}$ intravenously) every 3 weeks for six cycles and FAC (fluorouracil $500 \mathrm{mg} / \mathrm{m}^{2}$ intravenously, adriamycin $50 \mathrm{mg} / \mathrm{m}^{2}$ intravenously, cyclophosphamide $500 \mathrm{mg} / \mathrm{m}^{2}$ intravenously every 3 weeks for 6 cycles).

\section{Cognitive assessment}

The self-reported English version of the Fact-Cog (Version 3) was used to assess participants for chemobrain symptoms. The researcher tactfully assessed the participants' English proficiency by randomly asking them a set of questions based on their comprehension of the participants' information leaflet. The Fact-Cog instrument is a questionnaire with four subscales - perceived cognitive impairment (CogPCI), perceived cognitive ability (CogPCA), noticeability (CogOth) and impact on quality of life (CogQoL) - and it is used to measure each participant's cognitive function throughout the course of chemotherapy (Park et al. 2019). This instrument includes 37 items that assess verbal fluency, concentration, memory, functional interference, mental acuity and multitasking ability (Vega et al. 2019). This study focused on the perceived cognitive impairment (range 0-72). A 5 -point Likert scale ( $0=$ never to $4=$ several times a day) is used to rate each of the items over a 7-day period. Negatively and positively worded items are included in order to assess cognitive function. Higher scores on negatively worded statements indicate greater severity of cognitive impairment, whilst higher scores of positively worded ones indicate good cognitive function. On memory, for example, higher scores indicate better memory function. The Cronbach's alpha (scale reliability) value for this subscale was 0.83 which demonstrates its acceptable reliability (Victorson et al. 2008).

\section{Procedure and data collection}

Each participant was recruited with the assistance of the treating oncologist who made the diagnosis of breast cancer and determined the stages. The oncologist and/or the sister in-charge who were informed about the inclusion criteria of the study, assisted with the recruitment. After diagnosis and staging, either the oncologist or the sister-in-charge informed the patient about the study and explained what it involved. Those meeting the inclusion criteria were asked if they were interested in participating in the study. Patients who agreed were asked to wait in the waiting room in the breast cancer clinic, thereafter the researcher was introduced to the patient by the sister. The researcher verified that the patient met the inclusion criteria; thereafter he provided informed oral and written information about the study. Prior to receiving informed written consent and commencement of data collection, it was emphasised that the patient had the right to withdraw from the study at any point and/or refuse to participate in the study, without consequences for continued care at the clinic. Data collection involved completing a 5-min socio-demographic-health questionnaire at baseline, and a 10-min self-reported Fact-Cog measure at three points (baseline $\left[\mathrm{T}_{0}\right]$, third cycle $\left[\mathrm{T}_{1}\right]$, sixth cycle $\left[\mathrm{T}_{2}\right]$ ) during the participants' scheduled visits to the breast clinic. Cognitive assessment of participants was done just after cancer staging and prior to commencement of chemotherapy (baseline $\left[\mathrm{T}_{0}\right]$ ), midway through chemotherapy (third cycle $\left[\mathrm{T}_{1}\right]$ ) and at the completion of chemotherapy (sixth cycle $\left[\mathrm{T}_{2}\right]$ ). Written informed consent was obtained from all the participants prior to the assessments.

\section{Ethical considerations}

This study was approved by the Sefako Makgatho Health Sciences University Research and Ethics Committee (Protocol Number: /M/194/2018: PG) and the clinical director of Dr George Mukhari Academic Hospital. Participation in the study was voluntary and all patients had to provide informed consent. As part of the informed consent process, each of the participants was provided with detailed information about the study. It was also emphasised to them that they had the right to refuse to participate or to withdraw from the study without any consequences to their continued care. It was also clarified to each participant that the researcher does not form 
TABLE 1: Characteristics of participants.

\begin{tabular}{|c|c|c|c|c|c|}
\hline Variable & CMF regimen & $\%$ & FAC regimen & $\%$ & $p$ \\
\hline Mean age & 53.80 & - & 48.05 & - & $0.05^{*}$ \\
\hline Age range & - & - & - & - & 0.12 \\
\hline $31-50$ & 02 & 20 & 11 & 55 & \\
\hline $51-60$ & 08 & 80 & 09 & 45 & \\
\hline \multicolumn{6}{|l|}{ Ethnic group } \\
\hline Black African & 10 & 33 & 20 & 67 & \\
\hline Marital status & - & - & - & - & - \\
\hline Married & 04 & 40 & 08 & 40 & \\
\hline Divorced & 02 & 20 & 03 & 15 & \\
\hline Separated & 00 & 00 & 01 & 05 & \\
\hline Widowed & 01 & 10 & 05 & 25 & \\
\hline Single & 03 & 30 & 03 & 15 & \\
\hline Employment status & - & - & - & - & - \\
\hline Unemployed & 07 & 70 & 12 & 60 & \\
\hline Full-time & 02 & 20 & 02 & 10 & \\
\hline Part-time & 01 & 10 & 06 & 30 & \\
\hline Educational level & - & - & - & - & 0.15 \\
\hline Lower primary & 00 & 00 & 00 & 00 & \\
\hline Primary & 01 & 10 & 01 & 05 & \\
\hline Middle school & 03 & 30 & 05 & 25 & \\
\hline High school & 04 & 40 & 14 & 70 & \\
\hline Post school & 02 & 20 & 00 & 00 & \\
\hline Breast cancer stage & - & - & - & - & 1.00 \\
\hline 11 & 04 & 40 & 08 & 40 & \\
\hline III & 06 & 60 & 12 & 60 & \\
\hline
\end{tabular}

CMF, Cyclophosphamide, methotrexate, fluorouracil; FAC, fluorouracil, adriamycin cyclophosphamide.

Fisher exact tests.

$*$, Significance level set as $p<0.05$.

part of the treatment team and that information gathered during the study will be treated as confidential and will not be shared with others unless indicated by the participant. The participants were also informed that any personal information will be anonymised.

\section{Statistical analysis}

Descriptive statistics (i.e. means, standard deviation [SD], etc.) were performed on the participant's health and demographic information. The Fact-Cog scores for each treatment group were calculated. Pre-, during, and post-chemotherapy mean Fact-Cog scores were compared by paired $t$-tests and the nonparametric Wilcoxon signed-rank test. Differences between pre-, during and post-chemotherapy scores were analysed. All statistical analyses were performed on Statistical Analysis System (SAS Institute Inc., Carey, NC, USA), Release 9.4 or higher, running under Microsoft Windows for a personal computer. All tests were two-tailed and held statistical significance at $p<0.05$.

\section{Results}

\section{Participant characteristics}

Of the participants, 33\% $(n=10)$ were assigned to a chemotherapy regimen comprising of CMF, whilst $67 \%$ $(n=20)$ were treated with an FAC regimen. The mean age of the total participants was 50 years $(N=30)$, whilst that of the CMF group was 54 years and that of the FAC group was 48 years. The majority of the 30 participants had at least a
TABLE 2: Comparison of subjective self-reported cognitive function with chemotherapy regimens (cyclophosphamide, methotrexate, fluorouracil and fluorouracil, adriamycin, cyclophosphamide).

\begin{tabular}{|c|c|c|c|c|c|c|c|c|c|c|}
\hline \multirow[t]{2}{*}{ Variable } & \multicolumn{5}{|c|}{ CMF } & \multicolumn{5}{|c|}{ FAC } \\
\hline & $N$ & $M$ & SD & $p$ & $t$ & $N$ & $M$ & SD & $p$ & $t$ \\
\hline $\mathrm{T}_{0}$ & 10 & 57.20 & 4.32 & $<0.0001$ & - & 20 & 56.45 & 3.36 & $<0.0001$ & - \\
\hline $\mathrm{T}_{1}$ & 10 & 50.90 & 7.50 & $<0.0001$ & - & 20 & 49.00 & 7.61 & $<0.0001$ & - \\
\hline $\mathrm{T}_{2}$ & 10 & 46.80 & 12.00 & $<0.0001$ & - & 20 & 43.90 & 12.88 & $<0.0001$ & - \\
\hline$T_{0}-T_{1}$ & 10 & 6.30 & 6.24 & $0.011 *$ & 3.19 & 20 & 7.45 & 6.90 & $0.0001 *$ & 4.83 \\
\hline$T_{0}-T_{2}$ & 10 & 10.40 & 11.31 & $0.017^{*}$ & 2.91 & 20 & 12.55 & 12.05 & $0.0002 *$ & 4.66 \\
\hline
\end{tabular}

Note: Differences in subjective cognitive function between chemotherapy regimens were compared using paired sample $t$-tests and Fisher exact tests.

CMF, Cyclophosphamide, methotrexate, fluorouracil; FAC, fluorouracil, adriamycin cyclophosphamide; $T_{0}$, baseline; $T_{1}$, cycle $3 ; T_{2}$, cycle $6 ; S D$, standard deviation.

$*$, Significance level set as $p<0.05$.

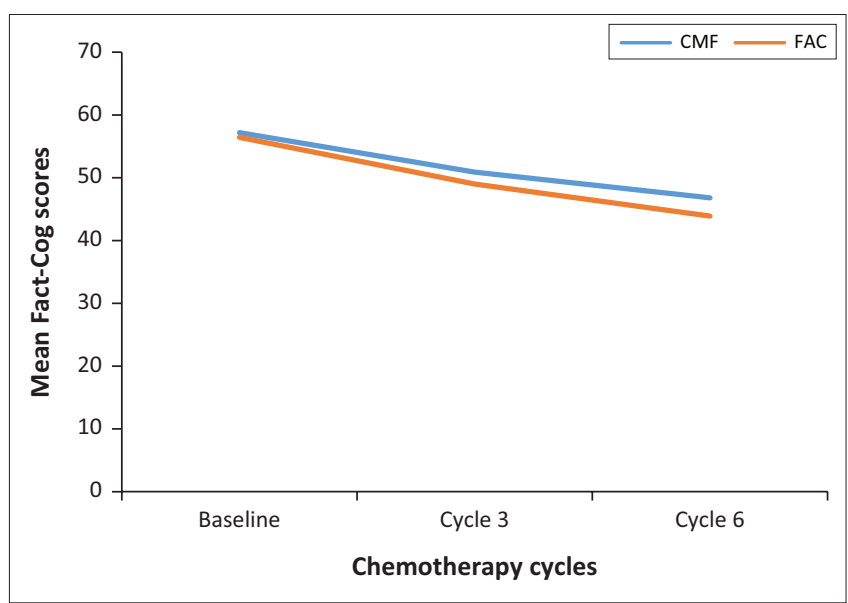

Note: Cognitive performance from baseline to completion of chemotherapy: CMF (baseline/ cycle 3: $M$ difference $=6.30, p<0.05$; baseline/cycle 6: $M$ difference $=10.40, p<0.05$ ) and FAC (baseline/cycle 3: $M$ difference $=7.45, p<0.05$ ); baseline $/$ cycle 6: $M$ difference $=12.55$, $p<0.05)$.

CMF, Cyclophosphamide, methotrexate, fluorouracil; FAC, fluorouracil, adriamycin, cyclophosphamide; FACT-Cog, Functional Assessment of Cancer Therapy-Cognition.

*, Significance level set as $p<0.05$

FIGURE 1: Perceived cognitive function and chemotherapy.

high school educational qualification (18; 60\%), whilst $(19 ; 63 \%)$ were unemployed (see Table 1$)$.

\section{Chemotherapy-related subjective cognitive impairment}

Of a total of 30 participants, 33\% in the CMF group and $67 \%$ in the FAC group reported significant perceived cognitive impairment $(p<0.05)$. In the CMF group, there was significant change in the perceived cognitive function from baseline $(M=57.20, \mathrm{SD}=4.32)$ to $\mathrm{T}_{2}$ (cycle 6) $(M=46.80, \mathrm{SD}=12.00), t(9)=2.91, p=0.017$ (see Table 2$)$. Similarly, the FAC group demonstrated a significant decline in perceived cognitive function from baseline $(M=56.45, \mathrm{SD}=3.36)$ to $\mathrm{T}_{2}($ cycle 6$)(M=43.90, \mathrm{SD}=12.88)$, $t(19)=4.66, p<0.0002$. The participants on the FAC regimen $(M=12.55, \mathrm{SD}=12.05)$ compared to the participants on the $\mathrm{CMF}$ regimen $(M=10.40, \mathrm{SD}=11.31)$ demonstrated greater perceived cognitive change from baseline to $T_{2}$ (cycle 6) (see Table 2). The change was however not statistically significant $(t[28]=0.47, p=0.642)$. Whilst these changes are not significant, they are still clinically meaningful, given that the FAC group showed greater perceived cognitive decline compared to the CMF group throughout the treatment (see Figure 1). 


\section{Discussion}

The present study found that patients with breast cancer (stages II and III) had a decline in Fact-Cog scores from baseline $\left(\mathrm{T}_{0}\right)$ to completion of chemotherapy $\left(\mathrm{T}_{2}\right)$. These results of subjective cognitive complaints are consistent with previous studies which showed that patients with breast cancer are at high-risk for chemotherapy-related cognitive impairment (CRCI) (Jansen et al. 2011; Jim et al. 2012; Kohli et al. 2007). Noticeably, over the entire study, the patients showed a decline in Fact-Cog scores in both the CMF and FAC chemotherapy regimen groups. These results concur with existing research which showed that the administration of multiple cytotoxic agents such as CMF and FAC can lead to cognitive decline (Cheung, Chui \& Chan 2012; Verrill 2009). These results are also consistent with evidence from a study that evaluated 42 patients with stage II and stage III breast cancer. The researchers found that in comparison to the nonchemotherapy group, the participants on chemotherapy (CMF and FAC) performed significantly worse on attention, memory and executive function tests $(p<0.05)$ (Chen et al. 2014).

Whilst both chemotherapy regimens were associated with ongoing cognitive decline in this study, the FAC group performed slightly worse than the CMF group on the Fact-Cog. Consistent with the current literature, anthracycline-based chemotherapy (adriamycin in the case of our study) exhibited a particularly high potential to induce cognitive decline (Kesler \& Blayney 2016). This can potentially explain the results from our study. Other studies also highlighted increased cognitive impairment which was associated with the neurotoxic effects of the FAC regimen (Henderson 2015; Ramalho et al. 2017). Likewise, Schagen and Wefel (2013) found that chemotherapy induced-toxicity was associated with adverse short-term and long-term cognitive effects, based on both subjective-self reported and objective cognitive tests.

Whilst our study is in agreement with previous studies, what remain unclear are the mechanisms for the occurrence of CRCI. The perceived cognitive impairment related to chemotherapy appears to be subtle in some cases and similar results emerged in other studies when standard dose chemotherapy was administered to cancer patients (Pendergrass, Targum \& Harrison 2018; Pereira et al. 2015). Perhaps the finding from the current study that FAC is associated with more cognitive problems as compared to $\mathrm{CMF}$, could be explained in terms of anthracycline effects which have been associated with greater neuroinflammatory and neurotoxic consequences (Allen et al. 2019). Further, multicentre, prospective cohorts with a large longitudinal follow-up design to explore the effects of anthracycline-based regimens are needed.

\section{Limitations of the study}

The study may have several potential limitations. Firstly, the small sample size could inhibit the generalisation of the findings around the effect of chemotherapy on subjective cognitive function of patients with breast cancer. Secondly, we only assessed subjective cognitive impairment and did not administer a battery of objective neuropsychological testing. Thirdly, in this study, only one subscale, which is cognition perceived cognitive impairment, was used and therefore aspects related to quality of life were excluded. Furthermore, the focus of this study was not to explore the underlying mechanism that can explain the observed cognitive decline in patients undergoing chemotherapy.

\section{Conclusion}

Although more work, which includes objective neurocognitive testing of larger samples of breast cancer patients, is needed to establish the full extent of cognitive change during and following chemotherapy, our preliminary results do suggest ongoing cognitive problems associated with chemotherapy in a small sample of women with stage II and stage III breast cancer, irrespective of the chemotherapy regimen. The perceived cognitive impairment observed in this study provides clinically meaningful information for the management of patients as greater perceived cognitive decline was demonstrated by the FAC group compared to the CMF group and this could assist clinicians to choose chemotherapy regimens with tolerable effects for their patients. Whilst cognitive deficits may be associated with chemotherapy-treated breast cancer patients and may impact on day-to-day functioning, it is not necessarily apparent. The study may, nevertheless, contribute to the documenting of the reality of chemobrain locally.

\section{Acknowledgements}

The authors would like to thank, with appreciation, the participants for their invaluable contribution to this study. We also acknowledge the work of Professor Hermanus Schoeman for the statistical analysis and Doctor Dikeledi Mokone for the recruitment of participants.

\section{Competing interests}

The authors declare that they have no financial or personal relationships that may have inappropriately influenced them in writing this article.

\section{Authors' contributions}

N.M.K and E.O. developed the research idea for this work and assisted with the pharmacology aspects, whilst A.G.L. worked on the psychological aspects. E.O. and A.G.L. supervised the project and all authors contributed to its writing and granted approval for its publication.

\section{Funding information}

This study was partially funded by the Sefako Makgatho Health Sciences University in Gauteng province of South Africa.

\section{Data availability}

The data that support the findings of this study are available from the corresponding author, N.M.K., upon reasonable request. 


\section{Disclaimer}

The views and opinions expressed in this article are those of the authors and do not necessarily reflect the official policy or position of any affiliated agency of the authors.

\section{References}

Akram, M., Iqbal, M., Daniyal, M. \& Khan, A.U., 2017, 'Awareness and current knowledge of breast cancer', Biological Research 50, 33. https://doi.org/10.1186/ s40659-017-0140-9

Allemani, C., Matsuda, T., Carlo, V.D., Harewood, R., Matz, M., Niksic, M. et al., 2018, 'Global surveillance of trends in cancer survival: Analysis of individual records fo $37,513,025$ patients diagnosed with one of 18 cancers during 2000-2014 from 322 population-based registries in 71 countries (CONCORD-3)', 391(10125), 1023-1075. https://doi.org/10.1016/S0140-6736(17)33326-3

Allen, B.D., Apodaca, L.A., Syage, A.R., Markarian, M., Baddour, A.A.D., Minasyan, H. et al., 2019, 'Attenuation of neuroinflammation reverses adriamycin-induced ct al., 2019, 'Attenuation of neuroinflammation reverses adriamycin-induced cognitive impairments', Acta Neuropatholog
https://doi.org/10.1186/s40478-019-0838-8

Bray, F., Ferlay, J., Soerjomataram, I., Siegel, R.L., Torre, L.A. \& Jemal, A., 2018, 'Global cancer statistics 2018: GLOBOCAN estimates of incidence and mortality worldwide for 36 cancers in 185 countries', CA: A Cancer Journal for Clinicians 68(6), 394-424. https://doi.org/10.3322/caac.21492

Chen, X., Li, J., Zhu, C., Li, D., Zhang, J. \& Wang, K., 2014, 'Cognitive function in breast cancer patients on chemotherapy: A longitudinal study', Zhonghua Yi Xue Za Zhi 94(1), 27-30.

Cheung, Y.T., Chui, W.K. \& Chan, A., 2012, 'Neuro-cognitive impairment in breast cancer patients: Pharmacological considerations', Critical Reviews in Oncology/ Hematology 83(1), 99-111. https://doi.org/10.1016/j.critrevonc.2011.09.001

DeSantis, C.E., Bray, F., Ferlay, J., Lortet-Tieulent, J., Anderson, B.O. \& Jemal, A., 2015 , 'International variation in female breast cancer incidence and mortality rates', Cancer Epidemiology and Prevention Biomarkers 24(10), 1495-1506. https://doi. org/10.1158/1055-9965.EPI-15-0535

Henderson, I.C., 2015, Breast cancer: Fundamentals of evidence-based disease management, Oxford University Press, viewed 22 September 2020, from https:// management, Oxford University Press, viewed 22 September 2020, from https:// oxfordmedicine.co

Isakoff, S.J., 2010, 'Triple negative breast cancer: Role of specific chemotherapy agents', Cancer Journal 16(1), 53-61. https://doi.org/10.1097/PPO.0b013e3181d24ff7

Janelsins, M.C., Mustian, K.M., Palesh, O.G., Mohile, S.G., Peppone, L.J., Sprod, L.K. et al., 2012 , 'Differential expression of cytokines in breast cancer patients receiving differen chemotherapies: Implications for cognitive impairment research', Supportive Care in Cancer 20(4), 831-839. https://doi.org/10.1007/s00520-011-1158-0

Jansen, C.E., Cooper, B.A., Dodd, M.J. \& Miaskowski, C.A., 2011, 'A prospective longitudinal study of chemotherapy-induced cognitive changes in breast cance patients', Supportive Care in Cancer: Official Journal of the Multinational Association of Supportive Care in Cancer 19(10), 1647-1656. https://doi. org/10.1007/s00520-010-0997-4

Jim, H.S.L., Phillips, K.M., Chait, S., Faul, L.A., Popa, M.A., Hussain, Y.-H.L.M.G. et al., 2012, 'Meta-analysis of cognitive functioning in breast cancer survivors previously treated with standard-dose chemotherapy', Journal of Clinical Oncology 30(29), 3578-3587. https://doi.org/10.1200/JCO.2011.39.5640

Kesler, S.R. \& Blayney, D.W., 2016, 'Neurotoxic effects of anthracycline- vs. nonanthracycline-based chemotherapy on cognition in breast cancer survivors, JAMA Oncology 2(2), 185-192. https://doi.org/10.1001/jamaoncol.2015.4333

Kohli, S., Griggs, J.J., Roscoe, J.A., Jean-Pierre, P., Bole, C., Mustian, K.M. et al., 2007, 'Self-reported cognitive impairment in patients with cancer', Journal of Oncology Practice 3(2), 54-59. https://doi.org/10.1200/JOP.0722001
Loh, K.P., Janelsins, M.C., Mohile, S.G., Holmes, H.M., Hsu, T., Inouye, S.K. et al., 2016, 'Chemotherapy-related cognitive impairment in older patients with cancer', Journal of Geriatric Oncology, 0(0). https://doi.org/10.1016/j.jgo.2016.04.008

Munir, F., Burrows, J., Yarker, J., Kalawsky, K. \& Bains, M., 2010, 'Women's perceptions of chemotherapy-induced cognitive side effects on work ability: A focus group study', Journal of Clinical Nursing, 19(9-10), 1362-1370. https://doi.org/10.1111/ j.1365-2702.2009.03006.x

Ono, M., Ogilvie, J.M., Wilson, J.S., Green, H.J., Chambers, S.K., Ownsworth, T. et al., 2015, 'A meta-analysis of cognitive impairment and decline associated with adjuvant chemotherapy in women with breast cancer', Frontiers in Oncology 5 , 59. https://doi.org/10.3389/fonc.2015.00059

Park, J.-H., Jung, Y.S., Jung, Y.-M. \& Bae, S.H., 2019, 'The role of depression in the relationship between cognitive decline and quality of life among breast cance patients', Supportive Care in Cancer 27(7), 2707-2714. https://doi.org/10.1007/ s00520-018-4546-x

Pendergrass, J.C., Targum, S.D. \& Harrison, J.E., 2018, 'Cognitive impairment associated with cancer', Innovations in Clinical Neuroscience 15(1-2), 36-44, viewed 21 October 2020, from https://www.ncbi.nlm.nih.gov/pmc/articles/ PMC5819720/.

Pereira, S., Fontes, F., Sonin, T., Dias, T., Fragoso, M., Castro-Lopes, J.M. et al., 2015, 'Neurological complications of breast cancer: A prospective cohort study', The Breast 24(5), 582-587. https://doi.org/10.1016/j.breast.2015.05.006

Ramalho, M., Fontes, F., Ruano, L., Pereira, S. \& Lunet, N., 2017, 'Cognitive impairment in the first year after breast cancer diagnosis: A prospective cohort study', The Breast 32, 173-178. https://doi.org/10.1016/j.breast.2017.01.018

Ribi, K., 2012, 'Cognitive complaints in women with breast cancer: Cross-cultural considerations', Annals of Oncology 23(10), 2475-2478. https://doi.org/10.1093/ annonc/mds182

Schagen, S.B. \& Wefel, J.S., 2013, 'Chemotherapy-related changes in cognitive functioning', EJC Supplements 11(2), 225-232. https://doi.org/10.1016/j. ejcsup.2013.07.007

Selamat, M.H., Loh, S.Y., Mackenzie, L. \& Vardy, J., 2014, 'Chemobrain experienced by breast cancer survivors: A meta-ethnography study investigating research and care implications', PLoS One 9(9), 108002. https://doi.org/10.1371/journal. pone.0108002

Simó, M., Rifa-Ros, X., Rodriguez-Fornells, A. \& Bruna, J., 2013, 'Chemobrain: A systematic review of structural and functional neuroimaging studies', Neuroscience \& Biobehavioral Reviews 37(8), 1311-1321. https://doi.org/10.1016/j. neubiorev.2013.04.015

Vardy, J., Wefel, J.S., Ahles, T., Tannock, I.F. \& Schagen, S.B., 2008, 'Cancer and cancertherapy related cognitive dysfunction: An international perspective from the Venice cognitive workshop', Annals of Oncology 19(4), 623-629. https://doi. org/10.1093/annonc/mdm500

Vega, J.N., Albert, K.M., Mayer, I.A., Taylor, W.D. \& Newhouse, P.A., 2019, 'Nicotinic treatment of post-chemotherapy subjective cognitive impairment: A pilot study', Journal of Cancer Survivorship: Research and Practice 13(5), 673-686. https://doi. Journal of Cancer Survivorship: Resear
org/10.1007/s11764-019-00786-6

Verrill, M., 2009, 'Chemotherapy for early-stage breast cancer: A brief history', British Journal of Cancer 101(1), S2-S5. https://doi.org/10.1038/sj.bjc.6605268

Victorson, D., Barocas, J., Song, J. \& Cella, D., 2008, 'Reliability across studies from the functional assessment of cancer therapy-general (FACT-G) and its subscales: A reliability generalization', Quality of Life Research: An International Journal of Quality of Life Aspects of Treatment, Care and Rehabilitation 17(9), 1137-1146. Quality of Life Aspects of Treatment, Care and
$\mathrm{https} / / / \mathrm{doi} .0 \mathrm{rg} / 10.1007 / \mathrm{s} 11136-008-9398-2$

Von Ah, D., Habermann, B., Carpenter, J.S. \& Schneider, B.L., 2013, 'Impact of perceived cognitive impairment in breast cancer survivors', European Journal of Oncology Nursing: The Official Journal of European Oncology Nursing Society 17(2), 236-241. https://doi.org/10.1016/j.ejon.2012.06.002

Weiss, H.D., Walker, M.D. \& Wiernik, P.H., 2010, Neurotoxicity of commonly used antineoplastic agents, Massachusetts Medical Society, Boston. 